\title{
Estimation of the Orientation of Vehicles in High-Resolution Radar Images
}

Fabian Roos, Dominik Kellner,

Jens Klappstein, Juergen Dickmann, Klaus Dietmayer, Klaus D. Müller-Glaser, and Christian Waldschmidt resale or redistribution to servers or lists, or reuse of any copyrighted component of this work in other works. 


\title{
Estimation of the Orientation of Vehicles in High-Resolution Radar Images
}

\author{
Fabian Roos*, Dominik Kellner ${ }^{\dagger}$, \\ Jens Klappstein ${ }^{\ddagger}$, Juergen Dickmann ${ }^{\ddagger}$, Klaus Dietmayer ${ }^{\dagger}$, Klaus D. Müller-Glaser ${ }^{\S}$, and Christian Waldschmidt* \\ *Institute of Microwave Techniques, University of Ulm, 89081 Ulm, Germany \\ ${ }^{\dagger}$ Institute of Measurement, Control and Microtechnology, University of Ulm, 89081 Ulm, Germany \\ ${ }^{\ddagger}$ Daimler AG, Group Research and Advanced Engineering, 89081 Ulm, Germany \\ $\S$ Institute for Information Processing Technologies, Karlsruhe Institute of Technology, 76049 Karlsruhe, Germany \\ Email: fabian.roos@uni-ulm.de
}

\begin{abstract}
The availability of high-resolution image radars allows estimating the orientation of vehicles from a single measurement without temporal filtering. This gives the opportunity to react even faster to certain critical traffic scenes. This paper presents an approach for estimating the orientation of a vehicle. The orientated bounding box algorithm known from literature is adapted to this end and a quality function is introduced to choose the optimal bounding box. In addition, a brute-force approach for determining the best possible outcome is presented.
\end{abstract}

\section{INTRODUCTION}

Driver assistance and safety systems are in high demand by customers and legislators. Those systems are working satisfactorally on highways, but for urban scenarios a more precise position, orientation and dimension estimation is essential for time crucial systems. It is assumed that the target vehicle does not drift, and hence the orientation can be used to determine the direction of movement.

\section{RELATED WORK}

Only single measurement methods are considered because the estimated orientation is subsequently used as input for more accurate and faster tracking not discussed in this paper. With increasing resolution, the radar reflections provide detailed contours of vehicles. To the best knowledge of the authors no work using radar has been published which estimates the orientation of an extended object using the reflections on the contour. Therefore methods used for processing laser scanner data and video images are considered as well.

\section{A. Boundary conditions for radar sensors and velocity vector}

The authors of [1] investigated the boundary conditions, which enables the estimation of the contour and thus the orientation of a vehicle using radar sensors. They suggest that a bandwidth larger than $1 \mathrm{GHz}$ and an angular resolution better than $1^{\circ}$ are necessary.

Assuming linear motion, e.g. the car is moving along a straight line, the authors of [2] present an approach to determine the velocity vector and the orientation. But an urban scenario contains turning vehicles, and estimating their movement is a key factor.

\section{B. Fitting models in laser scanner data}

Using a laser scanner, the contour of a vehicle can be extracted in detail. That is why an estimation of the orientation is possible. A common approach is to use (orientated) bounding boxes. For the determination one can use the convex hull. [3] introduces a simple and cost-effective procedure to find the minimum area rectangle of an enclosing convex hull using so called rotating calipers. [4] enhances this algorithm by introducing a symmetry assumption. The authors assume that a laser scanner can only detect the visible part of the contour and that the invisible part is symmetrical to the visible one. With this information a misaligned bounding box can often be avoided.

Another used model for vehicles consists of two perpendicular lines representing the most frequently seen two sides. Because of the orthogonality and one side being shorter than the other, this model is called L-fit. [5] presents an algorithm for this fit.

\section{Detecting perpendicular lines using a Hough transform}

A Hough transform could be used to detect rectangular shapes in video images. Depending on the model, two perpendicular lines or even a rectangle can be detected. [6] lists geometric relations which are necessary for the detection. [7] uses a laser rangefinder and a Hough transform.

\section{PRoblem Formulation}

The goal of this work is to obtain a robust algorithm which estimates the orientation of a vehicle from a single measurement. Using radars, it is important to use a robust approach, because effects such as multipath, clustering faults or clutter close to the target can cause detections outside the contour. If the viewing direction of the radar sensor is perpendicular to the contour of the vehicle, the reflections can outshine provoking also detections outside the contour.

As an example, in Fig. 1 the reflections of a vehicle at a distance of $12 \mathrm{~m}$ after applying an ordered statistic constant false alarm rate (OS-CFAR, [8]) and a clustering algorithm are shown. The coordinate system is an east-north-up reference frame, so the $x$-axis points in the driving direction and the $y$-axis is orientated to the left with respect to the driving 


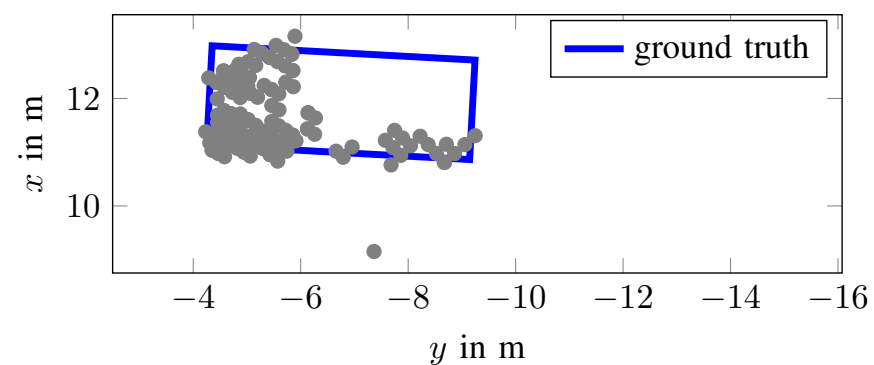

Fig. 1. Radar reflections of a vehicle in gray dots with the actual orientation as a ground truth. As a coordinate system the east-north-up reference frame is used.

direction. In this scenario the left corner is nearly on the right angle with respect to the sensor, so the radar reflections are strong. Also multipath reflection from the underbody can be seen. In addition, in front of the car there is a measured point, called outlier, which does not support the vehicular model. The algorithm should compensate such outliers so that the orientation is not flawed.

In contrast to the data from laser scanners, the captured contour of the vehicle has stronger deviations, e.g. it depends on the angle under which the vehicle can be seen and it can be cluttered. With the multipath reflections the symmetry assumption is no longer valid.

\section{Orientation Estimation Algorithm}

Every target vehicle consists of several received radar reflections. After the $O S-C F A R$ for detecting the reflections the clustering algorithm density based spatial clustering of applications with noise (DBSCAN, [9]) is used.

For each target, a number of clustered points exists in which a vehicular model is now fitted. The algorithm uses several iterations which result in different fitted models. For each iteration the current model must be evaluated, which is done by a quality function. As a comparative approach, a brute-force approach is presented using the quality function to determine the best possible estimation.

\section{A. Enhanced Orientated Bounding Box}

The presented algorithm is based upon the rotating calipers algorithm of [3]. The first step of this algorithm is to determine the convex hull of the points. With the extreme values of the $x$ and $y$-coordinates a basic rectangle is set up. Now the rectangle is rotated so that in each step one side of it is collinear with

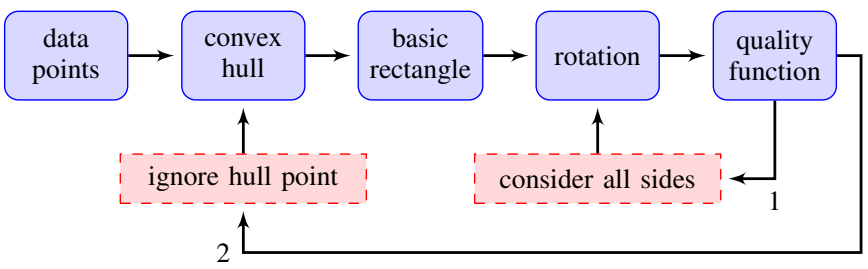

Fig. 2. Flow chart for the enhanced orientated bounding box algorithm.

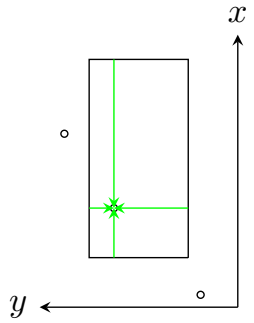

(a) The point inside the box is called inlier and supports the chosen model.
Fig. 3. Measuring the distance from a given point to the sides of the rectangle.

one side of the convex hull (see iteration loop 1 in Fig. 2). The number of necessary iterations are therefore directly associated with the number of convex hull points.

To enhance this approach and to consider outlier points as depicted in Fig. 1, each convex hull point will be ignored iteratively (see iteration loop 2 in Fig. 2). With one hull point left out, the rotated rectangles are once again considered.

The decision to ignore one hull point is chosen because in some cases it will not be sufficient to just ignore one outlier point. The reason is that erroneous detections occur in a number of points outside the vehicle and ignoring one single point has no significant effect on the convex hull.

\section{B. Evaluating each fit: the quality function}

Each fitted model of one iteration is evaluated using a quality function. Instead of minimising the area of the bounding box as proposed in [4], a set of factors are considered.

One important factor is the area of the resulting rectangle due to the fact that a misorientated bounding box usually needs more space than a correct one.

As can be seen in Fig. 1 a lot of points lie on the contour of the rectangle if it is aligned correctly. Measuring the distance from each point to the nearest appropriate side results in an optimisation variable which can be minimised as well. For points inside the rectangle, the smallest distance is taken as depicted in Fig. 3 (a). For outliers as in Fig. 3 (b) an incorrect procedure would be to select the minimum distance, because the distance to the right side would be taken as an optimisation variable. In fact the larger distance to the bottom of the rectangle has to be considered. Now it is opportune to increase the rectangle.

The points which support the chosen model lie inside the rectangle and are called inliers. All points outside the model are regarded as not supporting and classified as outliers. The relation between inliers and outliers is the third important factor.

In summary the area of the rectangle should be as small as possible, the distance of points to the respective side should be small and nearly all points should lie inside the rectangle.

\section{Brute-force approach as best case scenario}

The enhanced orientated bounding box algorithm (EOBB) is limited in the angular rotation steps given by the convex 


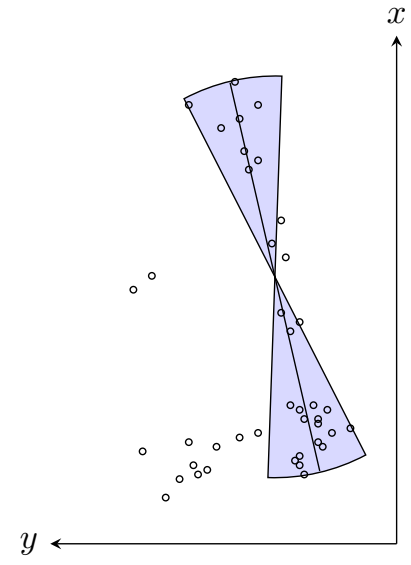

(a) The main direction is rotated to compensate a disadvantageous determination flawed by outliers.

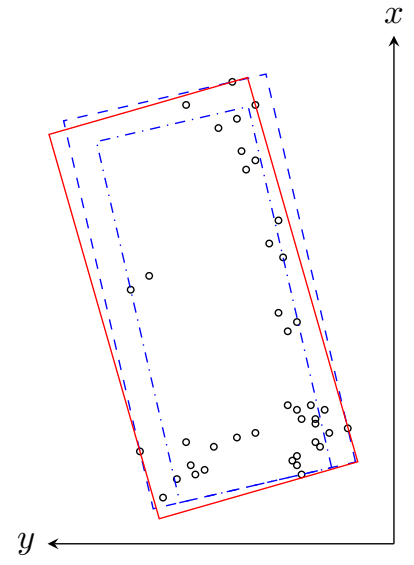

(b) Using the actual main direction the maximum area rectangle (dashed) is calculated and shrunk (dash-dotted). In addition a rectangle is constructed using a rotated direction (solid).
Fig. 4. Schematic of the brute-force approach. Iterating the main direction in (a) and expanding / shrinking of the rectangle in (b).

hull. Even with iteratively omitting one hull point the step size is still limited. To cope with this limitation the following algorithm is introduced.

Usually, one strong distinctive contour can be seen in the radar reflections. This is called the main direction of the radar reflections and is determined in the first step of the algorithm. Best results were obtained with the random sample consensus (RANSAC) algorithm introduced by [10]. The reflection points on the transition to the second detected contour shift the line a bit. Also the selected iteration need not be the idealistic one. To cope with this uncertainty, the main direction is rotated in both angular directions by several steps as depicted in Fig. 4 (a).

The maximum area rectangle including all points is set up for each main direction. In the next iteration steps, this rectangle is shrunk as shown in Fig. 4 (b). The basic rectangle is the dashed one, which is then shrunk, e.g. to the dash-dotted one. This is done for all rotated main directions. The solid rectangle is shown as an example of another direction. Each rectangle is evaluated with the quality function introduced in section IV-B.

This algorithm is obviously computationally expensive and therefore only used to show what possible best case scenario is achievable.

\section{EXPERIMENTAL RESULTS}

The presented algorithms are evaluated with a realistic measurement and compared to the orientated bounding box algorithm known in literature.

\section{A. Experimental setup and measurement}

Two experimental $77 \mathrm{GHz}$ radar sensors are mounted at the front of the vehicle. The bandwidth is $500 \mathrm{MHz}$, the angular resolution is $1^{\circ}$ and the field of view is $\pm 45^{\circ}$. The vehicle with the radar sensors and the target car are both equipped with
TABLE I

ALGORITHM COMPARISON

\begin{tabular}{l|r|c|r|c} 
Algorithm & mean in & median in $^{\circ}$ & rms in $^{\circ}$ & mae in \\
\hline OBB & 0.09 & -0.04 & 10.63 & 4.26 \\
OBB + qf & $\approx 0.00$ & -0.13 & 7.70 & 2.75 \\
EOBB & $\approx 0.00$ & -0.21 & 6.83 & 2.73 \\
MainDir & -0.19 & -0.19 & 5.36 & 2.34
\end{tabular}

an inertial measurement unit with differential GPS (DGPS) support to exactly determine the position and orientation of the cars for a precise error estimation of the presented algorithms.

The analysed scene consists of 2000 measurements with target distances from $8 \mathrm{~m}$ to $30 \mathrm{~m}$ while the target car is driving in circles and figure eights.

\section{B. Statistical error examination}

Each algorithm estimates the orientation of the vehicle labeled with $\varphi_{\text {est }}$. The ground truth is $\varphi_{\mathrm{GT}}$ and therefore the error is defined as $\varphi_{\mathrm{err}}:=\varphi_{\mathrm{est}}-\varphi_{\mathrm{GT}}$. This error is analysed with the following statistical parameters: the mean, the median, the root mean square (rms) and the introduced median absolute error which is defined by mae $=\operatorname{median}\left\{\operatorname{abs}\left(\varphi_{\mathrm{err}}\right)\right\}$.

In Table I those parameters are shown for the presented algorithms. The orientated bounding box $(O B B)$ algorithm from literature is susceptible to outlier points as there is no compensation implemented. With the introduction of a quality function $(O B B+q f)$, both rms and mae errors decrease by $28 \%$ and $35 \%$ respectively. Using the enhanced orientated bounding box $(E O B B)$, the rms error significantly decreases again by $13 \%$, whereas the mae is almost constant due to the correction of measurements with a large error resulting from outlier targets. Best results are achieved using the in section IV-C presented brute-force algorithm which determines the main direction (MainDir). The rms decreases by $22 \%$ and the mae by $14 \%$ respectively compared to $E O B B$ because this algorithm is not limited to the fixed angular steps resulting from the convex hull. Taking the needed calculation time into account, the brute-force approach is clearly more complex but the gain is limited.

If the absolute value is taken from all errors and then the median is calculated, which is the definition of mae, then $50 \%$ of the determined orientations using the $E O B B$ algorithm are better then $2.73^{\circ}$.

As can be seen from the values in Table I, all algorithms are bias free, so there is no preferred orientation. This is confirmed by the median, which is also nearly zero.

\section{Dependencies of the error}

The presented algorithms yield optimal results when two sides of the target vehicle are detected. Two stable contours offer the possibility for an ideal fit of a rectangle. To show this dependency the aspect angle is introduced as $\varphi_{\mathrm{asp}}:=\varphi_{\mathrm{GT}}-\varphi_{\mathrm{az}}$ at which $\varphi_{\mathrm{az}}$ is the azimuth angle under which the target vehicle is detected.

In the rear view with $\varphi_{\text {asp }}=0^{\circ}$ and the front view with $\varphi_{\text {asp }}=180^{\circ}$ only the short contour is directly visible to the 
radar and the orientation estimation is difficult. At $\varphi_{\text {asp }}=90^{\circ}$ the long side is detected and the errors decrease compared to the short side. The fewest errors occur if both sides are visible while the car is departing at $\varphi_{\text {asp }}=45^{\circ}$ or is approaching at $\varphi_{\text {asp }}=135^{\circ}$.

This aspect can be seen in Fig. 5 (a) for the $E O B B$. Additionally, the azimuth angle is shown as the color of the errors. If the target vehicle is at the edge of the field of view the errors are also increasing. In Fig. 5 (b) the dependency of the aspect angle for the different algorithms is shown. In Fig. 5 (c) the radar reflections do not allow a fitting, but in (d) the reflections are ideal resulting in a minor error.

\section{CONCLUSION}

An algorithm to estimate the orientation of a vehicle in high-resolution radar images is presented in this paper. The algorithm uses single measurements and is based on orientated bounding boxes which are selected using a quality function. Using the enhanced orientated bounding box in $50 \%$ of the measurements the orientation error is smaller than $2.73^{\circ}$. The root mean square error is $6.83^{\circ}$ for this measurement and $36 \%$ smaller compared to the orientated bounding box algorithm known in literature. Also, a brute-force algorithm is introduced to determine the best case scenario, which would result in $50 \%$ better results compared to the basic orientated bounding box algorithm. The fact that the calculation time is much larger must be considered. The improved outcome is due to the consideration of the possible outliers in radar data.

\section{REFERENCES}

[1] H.-L. Blöcher, M. Andres, C. Fischer, A. Sailer, M. Goppelt, and J. Dickmann, "Impact of system parameter selection on radar sensor performance in automotive applications," Advances in Radio Science, vol. 10 , pp. 33-37, 2012.

[2] D. Kellner, M. Barjenbruch, K. Dietmayer, J. Klappstein, and J. Dickmann, "Instantaneous Lateral Velocity Estimation of a Vehicle using Doppler Radar," in 16th International Conference on Information Fusion, July 2013, pp. 877-884.

[3] G. Toussaint, "Solving Geometric Problems with the Rotating Calipers," Proceedings of IEEE MELECON 83, pp. 10-02, 1983.

[4] P. Kmiotek and Y. Ruichek, "Representing and Tracking of Dynamics Objects using Oriented Bounding Box and Extended Kalman Filter," in Proceedings of the 11th International IEEE Conference on Intelligent Transportation Systems, 2008, pp. 322-328.

[5] H. G. Jung, Y. H. Cho, P. J. Yoon, and J. Kim, "Scanning Laser Radar-Based Target Position Designation for Parking Aid System," IEEE Transactions on Intelligent Transportation Systems, vol. 9, no. 3, pp. 406-424, 2008.

[6] C. R. Jung and R. Schramm, "Rectangle Detection based on a Windowed Hough Transform," in Proceedings of the 17th Brazilian Symposium on Computer Graphics and Image Processing, 2004, pp. 113-120.

[7] L. Zhao and C. Thorpe, "Qualitative and Quantitative Car Tracking from a Range Image Sequence," in Proceedings of IEEE Conference on Computer Vision and Pattern Recognition, 1998, pp. 496-501.

[8] H. Rohling, "Radar CFAR Thresholding in Clutter and Multiple Target Situations," IEEE Transactions on Aerospace and Electronic Systems, vol. AES-19, no. 4, pp. 608-621, July 1983.

[9] M. Ester, H.-P. Kriegel, J. Sander, and X. Xu, "A Density-Based Algorithm for Discovering Clusters in Large Spatial Databases with Noise," in Proceedings of 2nd International Conference on Knowledge Discovery and Data Mining. AAAI Press, 1996, pp. 226-231.

[10] M. A. Fischler and R. C. Bolles, "Random Sample Consensus: A Paradigm for Model Fitting with Applications to Image Analysis and Automated Cartography," Commun. ACM, vol. 24, no. 6, pp. 381-395, June 1981

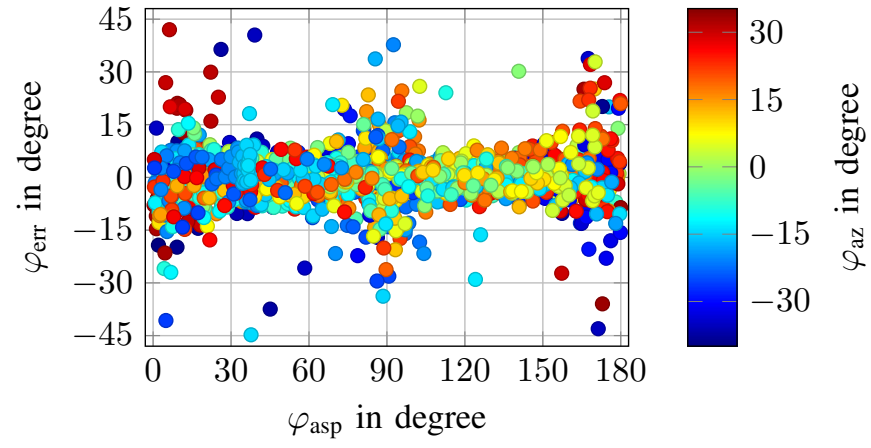

(a) Scatter plot of the error in the orientation estimation $\varphi_{\text {err }}$ plotted over the aspect angle $\varphi_{\text {asp }}$ and colored with the azimuth angle $\varphi_{\mathrm{az}}$ using the $E O B B$.

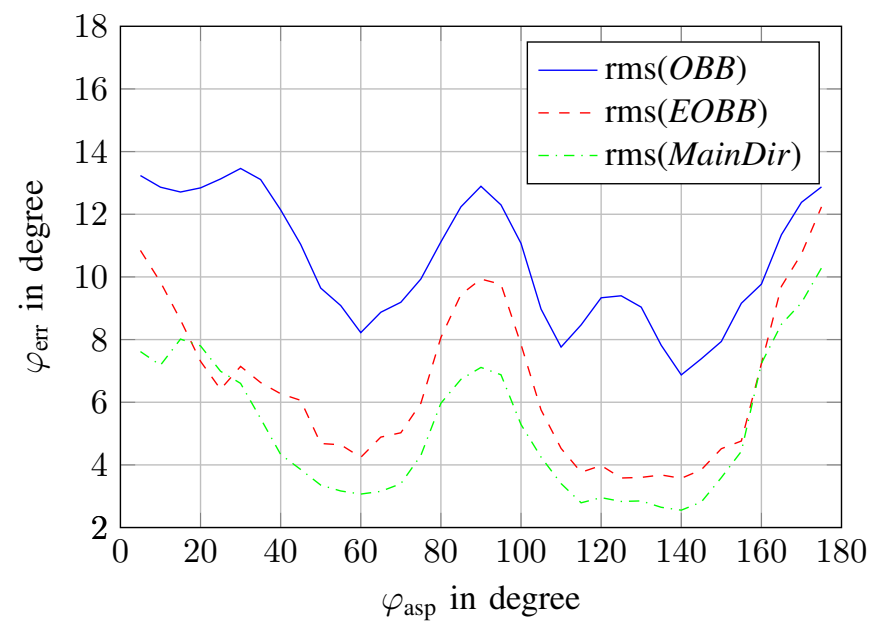

(b) Dependency of $\varphi_{\mathrm{err}}$ from $\varphi_{\mathrm{asp}}$ for different algorithms using the root mean square (rms) error.
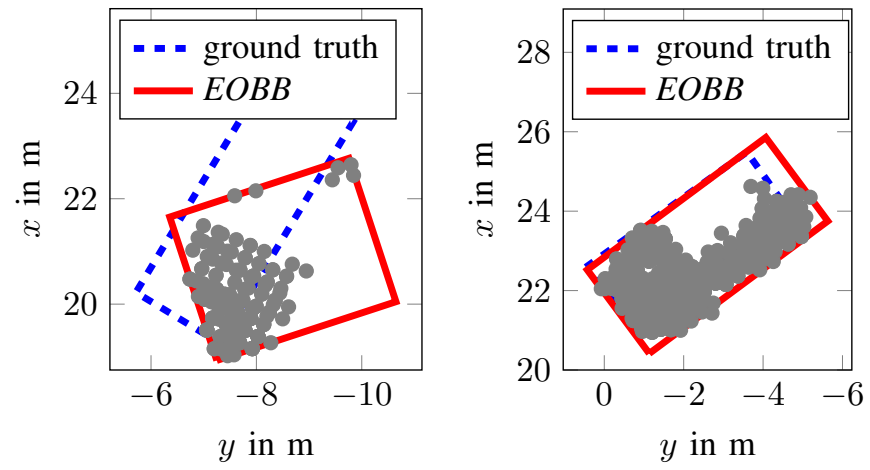

(c) Disadvantageous radar reflections, $\varphi_{\mathrm{err}}=-40.70^{\circ}, \varphi_{\mathrm{az}}=-26.22^{\circ}$

(d) Idealistic radar reflections, $\varphi_{\mathrm{err}}=0.98^{\circ}, \varphi_{\mathrm{az}}=-7.71^{\circ}$.
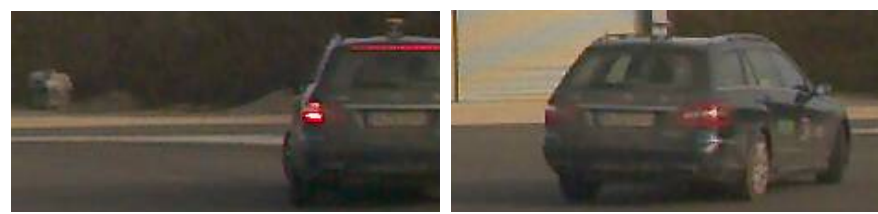

(e) Video image to (c), $\varphi_{\text {asp }}=5^{\circ}$.

(f) Video image to (d), $\varphi_{\text {asp }}=46^{\circ}$.

Fig. 5. Dependency of the orientation estimation error $\varphi_{\mathrm{err}}$ from the aspect angle $\varphi_{\text {asp }}$ and the azimuth angle $\varphi_{\mathrm{az}}$ in (a), comparing the algorithms using the rms in (b), exemplary radar reflections in (c) and (d) with corresponding video image in (e) and (f). 